\title{
A genome-wide association and meta-analysis reveal regions associated with seed size in cowpea [Vigna unguiculata (L.) Walp]
}

\author{
Sassoum Lo ${ }^{1}$ (1) María Muñoz-Amatriaín ${ }^{1,2}$ - Samuel A. Hokin ${ }^{3}$. Ndiaga Cisse ${ }^{4}$ Philip A. Roberts ${ }^{5}$. \\ Andrew D. Farmer ${ }^{3}$. Shizhong $\mathrm{Xu}^{1} \cdot$ Timothy J. Close ${ }^{1}$
}

Received: 21 March 2019 / Accepted: 24 July 2019 / Published online: 31 July 2019

(c) The Author(s) 2019

\begin{abstract}
Key message This paper combined GWAS, meta-analysis and sequence homology comparison with common bean to identify regions associated with seed size variation in domesticated cowpea.

Abstract Seed size is an important trait for yield and commercial value in dry-grain cowpea. Seed size varies widely among different cowpea accessions, and the genetic basis of such variation is not yet well understood. To better decipher the genetic basis of seed size, a genome-wide association study (GWAS) and meta-analysis were conducted on a panel of 368 cowpea diverse accessions from 51 countries. Four traits, including seed weight, length, width and density were evaluated across three locations. Using 51,128 single nucleotide polymorphisms covering the cowpea genome, 17 loci were identified for these traits. One locus was common to weight, width and length, suggesting pleiotropy. By integrating synteny-based analysis with common bean, six candidate genes (Vigun05g036000, Vigun05g039600, Vigun05g204200, Vigun08g217000, Vigun 1 I 187000 , and Vigun 1 g191300) which are implicated in multiple functional categories related to seed size such as endosperm development, embryo development, and cell elongation were identified. These results suggest that a combination of GWAS meta-analysis with synteny comparison in a related plant is an efficient approach to identify candidate gene (s) for complex traits in cowpea. The identified loci and candidate genes provide useful information for improving cowpea varieties and for molecular investigation of seed size.
\end{abstract}

\section{Introduction}

Cowpea [Vigna unguiculata (L.) Walp] is a multipurpose crop and a major source of dietary protein, fiber, vitamins,

Communicated by Heiko C. Becker.

Electronic supplementary material The online version of this article (https://doi.org/10.1007/s00122-019-03407-z) contains supplementary material, which is available to authorized users.

Sassoum Lo

slo008@ucr.edu

1 Department of Botany and Plant Sciences, University of California, Riverside, CA 92521, USA

2 Department of Soil and Crop Sciences, Colorado State University, Fort Collins, CO 80523, USA

3 National Center for Genome Resources, Santa Fe, NM 87505, USA

4 Centre d'Etude Régional pour l'Amélioration de l'Adaptation à la Sècheresse, ISRA/CERAAS, Thies, Senegal

5 Department of Nematology, University of California, Riverside, CA 92521, USA and minerals for millions of people and livestock in subSaharan Africa. Eaten in the form of fresh seeds, dry seeds, and fresh pods and as forage, cowpea is also an important crop in some parts of Asia, Latin America, and the USA (Dadson et al. 2005; Singh 2014). Cowpea is also an additional source of income for smallholder farmers in Africa, and the impact on household economies depends largely on seed appearance including seed size.

Since the beginning of agriculture, increased seed size has been a main domestication target as an important component of grain yield. Domesticated crops produce larger seeds compared to their wild ancestors. Seed size has several agronomically important impacts. Lush and Wien (1980) reported that large seeded cowpea emerged earlier than small seeded types when planted deeply (up to $5 \mathrm{~cm}$ ) and produce larger plants during early development. Cowpea seed size is an essential market trait in 
present-day Africa and other parts of the world. Consumers tend to prefer larger seeds (Mishili et al. 2009). Understanding the genetic basis of cowpea seed size is fundamental for breeding for this complex trait. Classical inheritance analysis suggested that at least eight loci control cowpea seed size (Drabo et al. 1984). Since the first publication of quantitative traits loci (QTLs) of seed weight by molecular markers (Fatokun et al. 1992), efforts have been made to identify QTLs associated with seed size based on seed mass using mapping populations with different genetic backgrounds (Andargie et al. 2014; Huynh et al. 2018; Lo et al. 2018; Lucas et al. 2013; Pan et al. 2017). All of these QTLs were discovered through linkage mapping using small populations. As a result, these QTLs are quite wide making the identification of causative genes challenging. Recently developed high-density genotyping tools and diverse germplasm subsets have made it possible to explore the genetic basis of this complex trait at finer resolution. To this aim, a genome-wide association study (GWAS) was conducted to investigate the genetic basis of seed size-related traits including seed length, width, and density using the recently developed genetic and genomic resources. Association mapping as described by Zhu et al. (2008) is a valuable tool to better understand the genetic basis of complex traits in plants. It has been widely adopted in several crop species to identify QTLs and to find candidate genes (Zhu et al. 2008). This approach enables the identification of genomic regions with finer resolution because of the smaller linkage disequilibrium in an association panel (Nordborg and Weigel 2008) as well as a larger population size.

A few GWA studies have been reported in cowpea for root architecture (Burridge et al. 2017), pod length (Xu et al. 2017), and black seed coat color (Herniter et al. 2018). No association mapping study has been reported on seed weight, length, width, and density in cowpea to date. Here, we conducted GWAS and meta-analysis to enhance our knowledge of the genetic architecture of seed size in cowpea. Meta-analysis (Rudner et al. 2002) is a useful tool through which GWAS results from different environments can be statistically pooled. This technique has been widely used in medical and social sciences. Seed size is known from studies on other plants to be controlled by various genes involved in different mechanisms including embryo and endosperm growth (Venglat et al. 2014). Genes affecting seed size have been cloned in Arabidopsis including APETALA2 (AP2), SHBI and IKU1 (Jofuku et al. 2005; Wang et al. 2010; Zhou et al. 2009). In soybean, GmCYP78A10 and GmCYP78A72 have been shown to play an important role in controlling seed size (Wang et al. 2015; Zhao et al. 2016). Here, we studied the genetic basis of seed size in domesticated cowpeas and identified single nucleotide polymorphisms (SNPs) significantly associated with seed sizerelated traits as well as promising candidate genes for which syntelogs in common bean (Phaseolus vulgaris) have been reported as seed weight candidate genes.

\section{Materials and methods}

\section{Plant materials}

A mini-core collection consisting of 368 accessions (MuñozAmatriaín et al. unpublished) was used for association mapping. The 368 accessions included landraces and breeding materials from 51 countries (Muñoz-Amatriaín et al. unpublished). It also included members of both the subspecies unguiculata and sesquipedalis (yardlong bean). The 368 lines were classified into six subgroups based on population structure (Muñoz-Amatriaín et al. unpublished). All accessions were grown under favorable conditions in the greenhouse and field in 2016 and 2017. Greenhouse growth was carried out at the University of California Riverside campus $\left(33.57^{\circ} \mathrm{N}\right.$; $117.20^{\circ} \mathrm{W}$ ) as follows: Seeds from each accession were grown in $3.8 \mathrm{~L}$ pots filled with $\mathrm{UCmix} 3$ with temperatures at $35^{\circ} \mathrm{C}$ day and $23{ }^{\circ} \mathrm{C}$ night. Mature, dried pods were harvested from each plant. For field experiments, each accession was planted in a single-row plot at the UCR Citrus Experiment Station, California, USA (UCR-CES, $33.97^{\circ} \mathrm{N}, 117.34^{\circ} \mathrm{W}$; Field11) and at the Coachella Valley Agricultural Research Station, California, USA (CVARS, $\left.33.52^{\circ} \mathrm{N}, 116.15^{\circ} \mathrm{W}\right)$. In field trials, mature, dried pods were harvested randomly as to minimize bias.

\section{Phenotypic evaluation and statistical analysis}

Seed size-related traits were evaluated as 100-seed weight (g), seed length $(\mathrm{mm})$, seed width $(\mathrm{mm})$, and seed density $\left(\mathrm{g} / \mathrm{cm}^{3}\right)$ in greenhouse and field experiments. The phenotyping for seed length and width was based on the average length and width of 10 seeds of each accession measured using a digital caliper. Seed density was calculated for each accession based on seed mass and its volume following the formula:

seed density $=\frac{\text { seed mass }}{\frac{\pi}{6} * \text { length } * \text { width }^{2}}$

Among the seed size traits, seed weight was measured in three environments. Broad sense heritability was estimated based on the data across the three environments. The variance components were estimated with the PROC VARCOMP procedure in SAS, and broad sense heritability calculated as follows:

$$
H^{2}=\frac{V_{\mathrm{G}}}{V_{\mathrm{G}}+V_{\mathrm{E}}}
$$


where $V_{\mathrm{G}}$ is the variance of genotype and $V_{\mathrm{E}}$ is the variance of the experimental error.

Pearson's correlation coefficients were calculated using the cor.test () function in R (Ihaka and Gentleman 1996). The correlation coefficient was calculated between seed mass, seed length, seed width, and seed density.

\section{SNP genotyping}

Total genomic DNA was extracted from dried leaves collected from one plant of each accession using Plant DNeasy (Qiagen, Germany). Total DNA was quantified using a Quant-IT dsDNA Assay Kit (Thermo Fisher Scientific, USA). The 368 accessions were genotyped using the Cowpea iSelect Consortium Array containing 51,128 SNPs (Muñoz-Amatriaín et al. 2017). Genotyping was conducted at the University of Southern California Molecular Genomics Core facility (Los Angeles, California, USA). SNPs were called using GenomeStudio software V.2011.1 (Illumina, Inc. San Diego, CA). For GWAS, data were filtered by removing SNPs with more than $25 \%$ missing calls, and minor allele frequency (MAF) less than 0.05 . The physical positions of these SNPs were determined using the IT97 K-499-35 reference genome (Lonardi et al. 2019).

\section{Genome-wide association study and meta-analysis}

GWAS was conducted on 100-seed weight (measured in three locations), length, width, and density (each measured in one location; CVARS). GWAS was performed using the mixed linear model (Zhang et al. 2010) in TASSEL V5.0 (http://www.maizegenetics.net/tassel). Principal component analysis (PCA) and a kinship coefficient matrix $(K)$ were generated in TASSEL. PCA was used to account for population structure $(Q)$, and $K$ was used to correct for relatedness of accessions. The percentage contribution of each SNP to the total phenotypic variation was calculated using marker $\mathrm{R}^{2}$ values (computed by TASSEL) multiplied by 100 .

Seed weight was measured in three environments. GWAS was first performed separately for each environment, and then results were combined via a meta-analysis. A simple meta-analysis procedure recommended by Kang et al. (2014) was used to increase power of association and detect $\mathrm{G} \times \mathrm{E}$ interaction loci. This method essentially treats multiple environments as multiple populations. Loci that have different effects across different environments are $\mathrm{G} \times \mathrm{E}$ interaction loci. The method has been used in detecting interaction loci in a multiple environment mouse experiment (Kang et al. 2014). Among several specialized algorithms, Fisher's method was adopted because the method only requires the $p$ values across multiple environments. However, this method requires a comprehensive tool to prove the distribution. Therefore, we developed a similar method that does not need the proof of the distribution. This method is called the Probit method. Let $p_{k}$ be the $p$ value from environment $k$ for $k=1, \ldots, m$, where $\mathrm{m}$ is the number of environments. The test statistic is defined as

$X=\sum_{k=1}^{m}\left[\Phi^{-1}\left(1-0.5 p_{k}\right)\right]^{2}$

where $\Phi^{-1}()$ is called the probit function, which is the inverse function of the standardized normal distribution. In other words, if $p=\Phi(z)$ and $p$ is a standardized uniform variable, then $z=\Phi^{-1}(p)$ is a standardized normal variable. The square of a standardized normal variable is a Chi-square variable with one degree of freedom. The sum of $m$ onedegree Chi-square variables is a Chi-square variable with $\mathrm{m}$ degrees of freedom. Under the null model ( $p_{k}$ is a standardized uniform variable between 0 and 1 ), this test statistic follows a Chi-square distribution with $m$ degrees of freedom (no proof is needed). The new $p$ value was calculated as follows:

$P_{\text {probit }}=1-\operatorname{Pr}\left(X_{m}^{2} \leq X^{2}\right)$

The result is much the same as in Fisher's method; the only difference is that the Chi-square distribution is given without the need for proof. The threshold for genome-wide significance cutoff was applied based on Bonferroni correction at $\alpha=0.05$.

\section{Candidate genes and syntelogs}

The regions significantly associated with the traits were localized on the cowpea reference genome (Lonardi et al. 2019) to determine the underlying candidate genes. The significant regions were also compared to syntenic regions on the common bean genome (Schmutz et al. 2014) using the legumeinfo.org instance of the Genome Context Viewer (GCV) (Cleary and Farmer 2017) to determine a list of common bean genes (syntelogs). Synteny relationships between genes were based on their assignment to the same gene families, using hmmsearch at a threshold of $1 \mathrm{e}^{-10}$ against Hidden Markov Models representing legume gene families https://legumeinfo.org/data/public/Gene_families/legum e.genefam.fam1.M65K; we also required them to occur in regions of similar genic content (10 or more homologous genes with conserved order in a neighborhood of 20 genes surrounding the candidate). Using functions of cowpeamine and legumemine (https://mines.legumeinfo.org), syntelogs were further intersected with a list of genes associated with seed weight as reported in Schmutz et al. (2014) based on their homology to functionally characterized Arabidopsis genes. Cowpea genes homologous to the common bean 
genes present in the intersection were considered to be strong candidate genes associated with seed weight.

\section{Results}

\section{Phenotypic variation and seed size trait correlations}

The phenotyping panel contained 368 cowpea accessions comprising both landraces and breeding materials, classified into six subgroups based on population structure (Muñoz-Amatriaín et al. unpublished). Seed size-related traits were evaluated based on seed weight, length, width and density. Mean and standard deviation were determined for each trait (Table 1). Broad sense heritability of seed weight was calculated, and the estimated value of 0.61

Table 1 Mean and standard deviation of the seed size traits in the panel

\begin{tabular}{llrl}
\hline Trait & Location & Mean & $\begin{array}{l}\text { Standard } \\
\text { deviation }\end{array}$ \\
\hline Seed weight $(\mathrm{g})$ & GH & 15.22 & 4.85 \\
& UCR-CES & 17.49 & 5.34 \\
& CVARS & 14.35 & 4.75 \\
Seed length $(\mathrm{mm})$ & UCR-CES & 6.59 & 1.36 \\
Seed width $(\mathrm{mm})$ & UCR-CES & 4.41 & 0.91 \\
Seed density $\left(\mathrm{g} / \mathrm{cm}^{3}\right)$ & UCR-CES & 2.27 & 0.83 \\
\hline
\end{tabular}

suggests relatively high heritability. The 368 accessions show a variation of grain sizes ranging from 5 to $31 \mathrm{~g}$ per 100 seeds. Principal component analysis (PCA) showed the distribution of "seed weight" (Fig. 1) in the diversity panel. The frequency distribution of seed weight is also shown in Supplemental Figure 1. To see the relationship between seed size traits, Pearson's correlation coefficients were calculated between seed mass, length, width, and density. Seed mass is highly correlated (positive) with seed length (0.84) and width (0.89). Furthermore, seed width and length showed moderate correlation (0.67), possibly because of the yardlong bean accessions in the diversity set. Negative correlations were observed between density and length $(-0.67)$, and density and width $(-0.85)$ indicating that as seeds are larger they are also less dense.

\section{Association mapping}

A total of 42,711 polymorphic SNPs were used for GWAS. A total of 17 loci were identified for the seed size-related traits (Fig. 2; Tables 2, 3). A hot spot was identified on chromosome Vu03, where QTLs for seed weight (Sw3.2), width (Swi3) and length coincided. Colocalization of seed size-related trait QTLs suggests pleiotropy or physical linkage of genes controlling seed size in cowpea.

\section{Distribution of seed weight}

$<10 \mathrm{~g} / 100$ seeds

$10-20 \mathrm{~g} / 100$ seeds

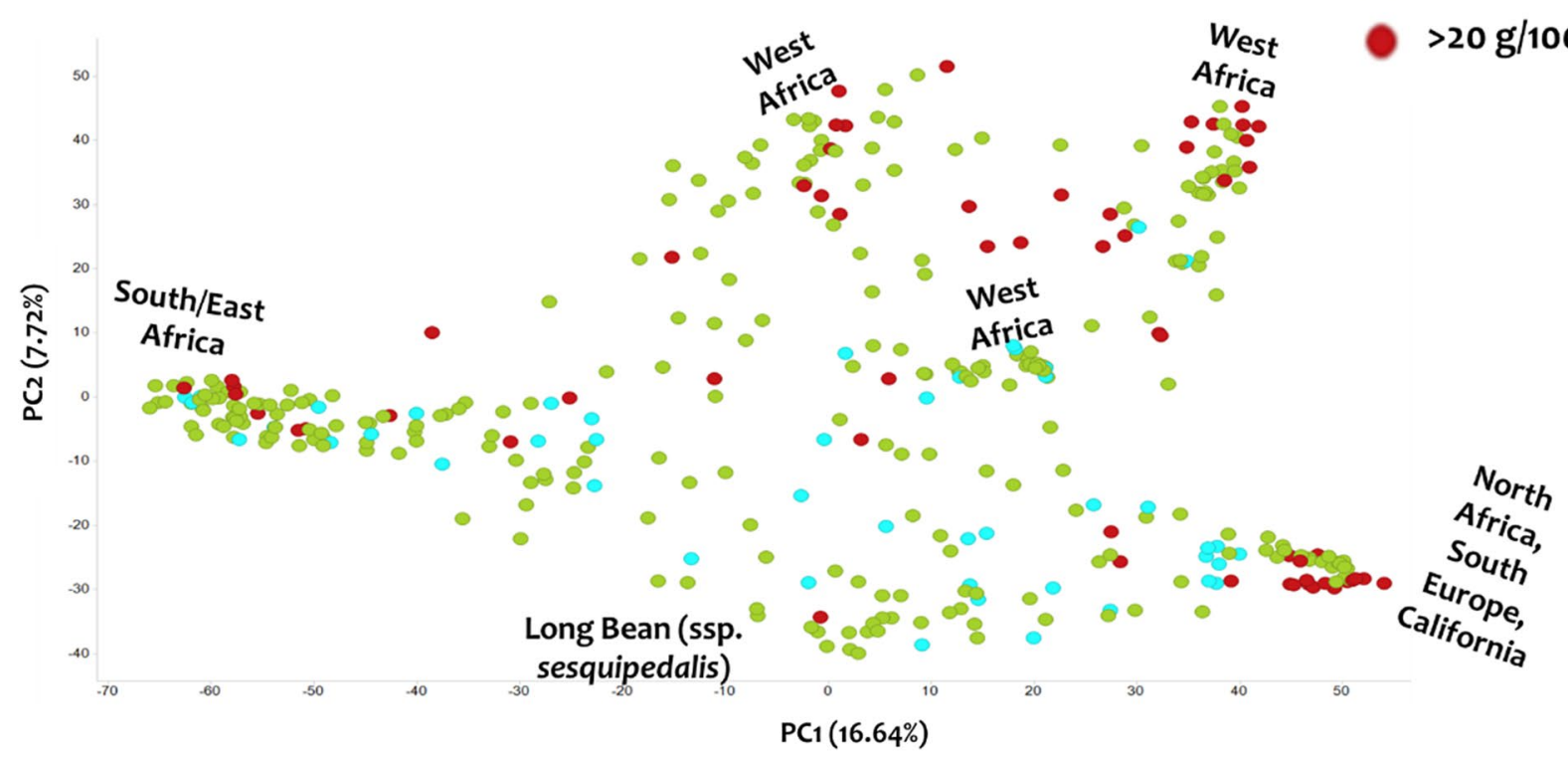

Fig. 1 Principal component analysis of the 368 accessions colored by seed weight range 


\section{Seed weight}

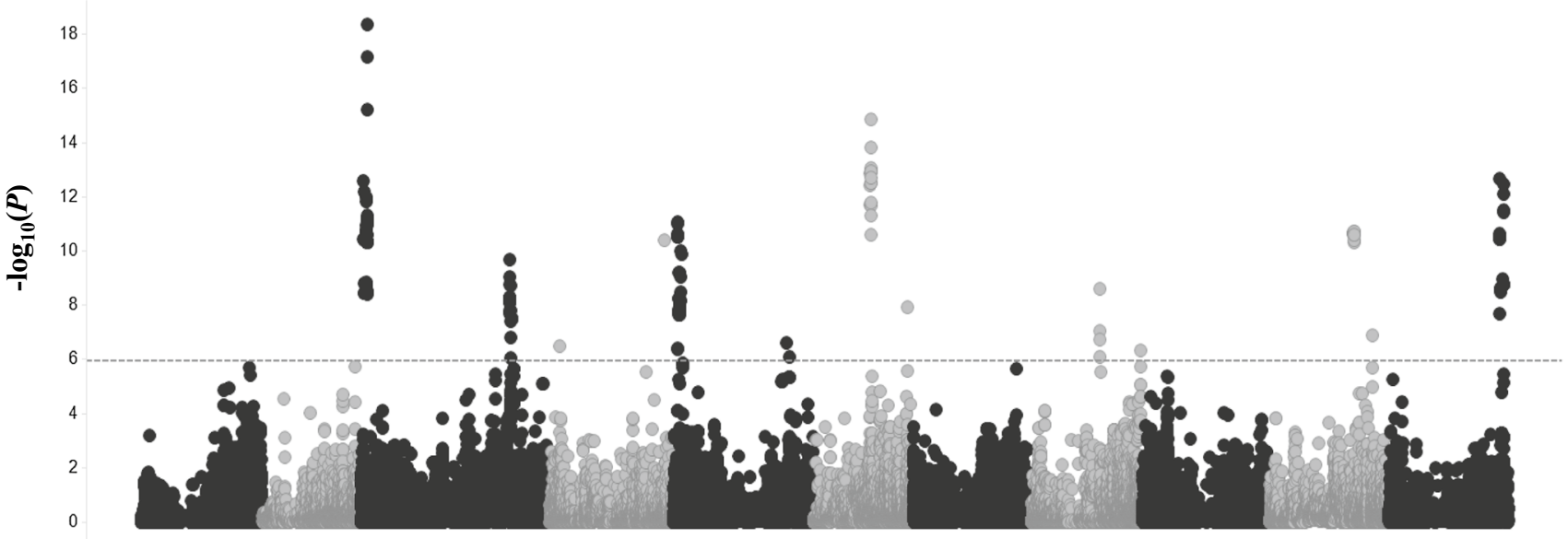

\section{Seed width}

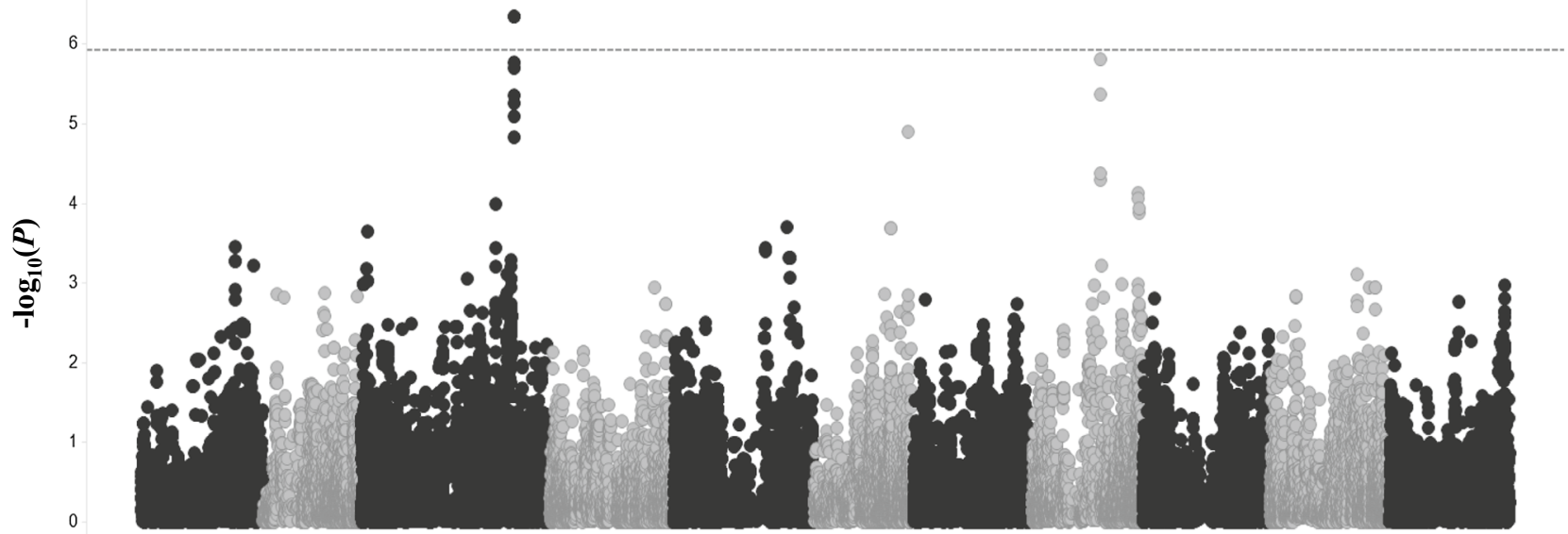

Seed density

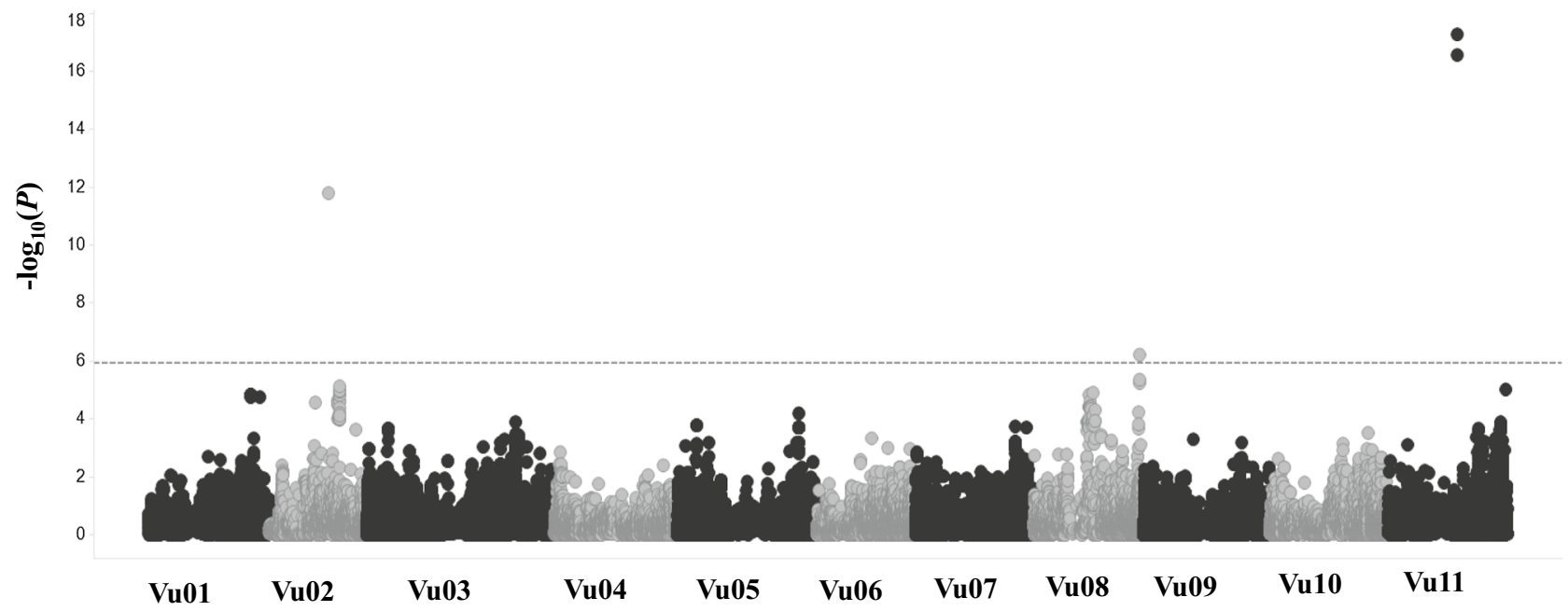

Fig. 2 Manhattan plot of GWAS meta-analysis for seed weight and GWAS for seed width and density. Negative $\log _{10} p$ values are plotted against physical position on each of the 11 chromosomes. Dashed line indicates Bonferroni-corrected genome-wide significance threshold 
Table 2 List of significant QTLs for seed weight from GWAS meta-analysis results

\begin{tabular}{llclcl}
\hline QTL & Peak SNP & Chr. & Position & $-\log _{10} p$ & Comment \\
\hline Sw3.1 & 2_26016 & 3 & 2223359 & 18.36 & GH, UCR-CES, CVARS, Meta \\
Sw3.2 & 2_40922 & 3 & 51562014 & 9.67 & UCR-CES, Meta \\
Sw4.1 & 2_14347 & 4 & 3483218 & 6.51 & Meta \\
Sw4.2 & 2_25894 & 4 & 39757606 & 10.41 & CVARS, Meta \\
Sw5.1 & 2_07617 & 5 & 1783613 & 11.08 & UCR-CES, CVARS, Meta \\
Sw5.2 & 2_08022 & 5 & 39230104 & 6.63 & UCR-CES, Meta \\
Sw6.1 & 2_07078 & 6 & 19698161 & 14.84 & GH, UCR-CES, CVARS, Meta \\
Sw6.2 & 2_07358 & 6 & 32476947 & 7.92 & Meta \\
Sw8.1 & 2_50483 & 8 & 23709814 & 8.61 & Meta \\
Sw8.2 & 2_17605 & 8 & 37710852 & 6.32 & Meta \\
Sw10.1 & 2_50509 & 10 & 29351777 & 10.73 & CVARS, Meta \\
Sw10.2 & 2_08400 & 10 & 35932948 & 6.87 & UCR-CES, Meta \\
Sw11 & 2_28551 & 11 & 38568662 & 12.69 & GH, UCR-CES, CVARS, Meta \\
\hline
\end{tabular}

Table 3 List of significant QTLs for seed width and density from GWAS results

\begin{tabular}{lllllrrrr}
\hline Trait & QTL & Peak SNP & Chr. & Position & $-\log _{10} p$ & $R^{2}(\%)$ & Alleles & Effect \\
\hline Seed width & Swi3 & 2_29499 & 3 & 52931236 & 6.35 & 7.09 & A/C & -0.59 \\
Seed density & Sd2 & $2 \_09625$ & 2 & 20857395 & 11.82 & 14.84 & G/T & 0.06 \\
& Sd8 & 2_27144 & 8 & 36984438 & 6.22 & 7.06 & A/C & -0.63 \\
& Sd11 & 2_41753 & 11 & 24148832 & 17.27 & 21.93 & C/T & 0.12 \\
\hline
\end{tabular}

\section{Seed weight}

GWAS was performed separately for the three environments, and results were combined via the Probit metaanalysis (see "Materials and methods" section). The metaanalysis identified 13 significant regions on chromosomes 3, 4, 5, 6, 8, 10, and 11 (Fig. 2; Table 2; Supplemental Table 1). Those loci included all the loci identified in each single environment (Supplemental Table 1), plus four loci that were not detected in any single environment. Four loci were consistent across two environments (UCR-CES and CVARS), while three loci were common to all three environments (GH, UCR-CES and CVARS), suggesting a presence of a significant $\mathrm{G} \times \mathrm{E}$ interaction. Effects and $R^{2}$ values of significant SNPs were calculated for each single environment and are reported in Supplemental Table 1. We also used Fisher's meta-analysis to combine results of the three replications for seed weight. The results are almost indistinguishable from the Probit method developed here (see "Materials and methods" section). The results of both methods are shown in Supplemental Table 2. Recall that four additional loci were detected by the meta-analysis. The effects of the four loci may not be sufficiently large to be detected in any single environment, but when results from individual environments were pooled via the metaanalysis, they were significant.

\section{Seed length, width, and density}

One significant region was associated with seed width on $\mathrm{Vu} 03$, and three regions were associated with seed density on Vu02, Vu08, and Vu11 (Fig. 2; Table 3; Supplemental Table 1). The strongest QTL (Sd11) was localized on Vu11 and explained the highest genetic variance $(21.51 \%$; Table 3; Supplemental Table 1). Significant loci for seed length were not identified, although three loci were detected slightly below the significance threshold. Since the Bonferroni-corrected threshold is very conservative, loci with levels slightly lower than the significance threshold are listed in Supplemental Table 1. Of the loci considered for seed length, one was common with the locus detected for seed weight (Sw3.2) and seed width (Swi3) on Vu03, and another one was common with the locus detected for seed density (Sd8) on Vu08.

\section{Candidate genes and syntelogs}

All identified loci were aligned to the cowpea reference genome (Lonardi et al. 2019), and the underlying genes are listed in Supplemental Table 3. Synteny-based analysis performed with common bean using GCV (https://legumeinfo .org/lis_context_viewer) identified six syntelogs from the intersection with seed weight candidate genes reported by 
Schmutz et al. (2014). The six syntelogs along with their corresponding cowpea genes are given in Supplemental Table 4. The corresponding cowpea genes of the syntelogs include two for Sw5.1 (Vigun05g036000, Vigun05g039600), one for Sw5.2 (Vigun05g204200), one for Sw8.2 (Vigun08g217000), and two for Sw11 (Vigun11g187000, Vigun11g191300). The gene Vigun05g036000 is annotated as cell wall protein while Vigun05g039600 encodes a phosphate transporter PHO1. Vigun05g204200 is annotated as encoding a polycomb group protein FERTILIZATION-INDEPENDENT ENDOSPERM and Vigun08g217000 codes for a histidine kinase 2. The genes Vigun1 1 g187000 and Vigun11g191300 are annotated as WD repeat-containing protein 61-like isoform 1 and delta (24)-sterol reductase-like protein, respectively. As comparative genomics is an efficient approach, we draw attention to these six interesting candidate genes.

\section{Discussion}

Seed size is one of the key yield determinants. Understanding the underlying genetic factors of cowpea seed size can help breeders develop improved varieties with a range of seed sizes. Also, identifying genes that could control seed size variation in domesticated cowpea can provide important insights into the genetic basis of adaptation, as seed size has been recognized as an important contributor to adaptation in plants (Chapin III et al. 1993; Igea et al. 2017). Previous studies using mapping populations from different genetic backgrounds have identified QTLs for seed size based on seed mass (Andargie et al. 2014; Fatokun et al. 1992; Huynh et al. 2018; Lo et al. 2018; Lucas et al. 2013; Pan et al. 2017). However, these studies have provided limited information on potential candidate genes for seed size. In cowpea, determining the genetic basis of complex traits has become increasingly effective due to the availability of the genome sequence (Lonardi et al. 2019) and high-density genotyping (Muñoz-Amatriaín et al. 2017). As the next step to gain new knowledge on the genetic control of seed size, we used GWAS and meta-analysis to identify loci and candidate genes for seed size-related traits at a higher resolution that has been possible previously.

The meta-analysis identified 13 loci for seed weight (Fig. 2; Table 2) including four loci that were not detected in any single location (Table 2 ). The power of meta-analysis to identify additional loci in the combined data set that were not detected in the single environment due to insufficient power provides support for its utility. As has been shown in many studies in medical and social sciences, meta-analysis can overcome the limits of an individual environment by increasing the resolution power and reducing false-positive findings (Evangelou and Ioannidis 2013). It is well known that increasing sample size can increase statistical power and decrease Type 1 error (Wang and Xu 2019). Meta-analysis is much like combining multiple populations (environments) into a pooled data and thus serves to increase the sample size. Rather than pooling the original data, this meta-analysis pools the results (the $p$ values). This result also revealed a presence of a significant $G \times E$ interaction with some loci being location specific. The presence of $\mathrm{G} \times \mathrm{E}$ interaction loci noted here confirms the complexity of seed weight. Overlap between our meta-analysis result and previous studies was found. BLAST searches of RLFP probe sequences from Fatokun et al. (1992) against the reference genome sequence (Lonardi et al. 2019) revealed that the two QTLs identified by Fatokun et al. (1992) seem to correspond to Sw3.2 and Sw6.1. Furthermore, Fatokun et al. (1992) reported that one of the QTLs (the one corresponding to Sw3.2 here) is orthologous to a seed weight QTL in mung bean (Vigna radiata), suggesting that the genomic region has remained conserved through evolution. Also, Pan et al. (2017) reported a locus for grain weight which overlapped with Sw3.2. The location of QTL Sw8.2 on Vu08 overlapped with that identified from several independent studies (Huynh et al. 2018; Lo et al. 2018; Lucas et al. 2013). However, Sw6.2 on Vu06 is very close to a locus identified by Lo et al. (2018) for seed weight. This study may serve to refine this locus, or may indicate a novel locus. Sw3.1, Sw4.1, Sw4.2, Sw5.1, Sw5.2, Sw8.1, Sw10.1, Sw10.2, and Sw11 represent novel associated loci, further suggesting the complexity of seed size. Consistent with results from (Drabo et al. 1984), our study demonstrated that at least eight loci control cowpea seed size. Our meta-analysis result can guide the choice of QTL targeted for marker-assisted selection.

In this study, we also identified significant regions for seed width and density. The locus for seed width (Swi3) was in the same region as Sw3.2. Also, a clear peak for seed length was noted on the same genomic region. We note also that this region overlapped with a region identified for grain weight by Pan et al. (2017). Taken together with the high correlation between seed mass, width, and length, we hypothesize that this QTL has a pleiotropic effect. However, the possibility of coexistence of multiple genes should not be excluded due to the complexity of these traits. Additional studies are necessary to further explore any of these hypotheses. In addition to their contribution to yield, seed width, density, and length are important traits that have impact on the market value of cowpea. These QTLs will be a valuable resource for the improvement of cowpea.

We identified genes within these QTLs regions (Supplemental Table 3) using the annotated cowpea reference genome (Lonardi et al. 2019). Among the associated loci for seed weight, six particular candidate genes were identified based on comparative genomic analysis with common bean. Two interesting candidate genes for Sw5.1 were Vigun05g036000 and Vigun05g039600. The former encodes 
a cell wall protein, which was reportedly associated with seed size (Cheng et al. 1996; Jin et al. 2009; Weber et al. 1996). The latter, Vigun05g039600, encodes a phosphate transporter PHO1. In Arabidopsis, a PHO1 gene has been reported to be a positive regulator of seed development that affects both cell size and cell number (Zhou et al. 2009). Since several genetic pathways are known to control seed size, this gene is a promising candidate. Synteny-based analysis suggested that Vigun05g204200, which is a potential candidate for Sw5.2, is annotated as encoding a polycomb group protein FERTILIZATION-INDEPENDENT ENDOSPERM (FIE). FIE genes are involved in endosperm development (Ohad et al. 1996) and have been shown to regulate seed size (Folsom et al. 2014). The candidate gene for Sw8.2 was Vigun08g217000 which codes for a histidine kinase 2. Interestingly, Vigun08g217000 has been identified as potential candidate gene for increased organ size during cowpea domestication (Lonardi et al. 2019) and its Arabidopsis ortholog $A H K 2$ has been shown to regulate seed size (Bartrina et al. 2017; Riefler et al. 2006). Similarly, two candidate genes for Sw11 were determined: Vigun1 1 g187000 and Vigunl 1 191300. The former is annotated as WD repeat-containing protein 61 -like isoform 1 . WD repeat proteins are mainly involved in cellular processes including cell division (van Nocker and Ludwig 2003). In addition, the Arabidopsis gene AT2G34260, which encodes a WD repeat protein, is required for embryo and endosperm development (Bjerkan et al. 2012). Vigun1lg191300 encodes a delta (24)-sterol reductase protein and is an ortholog of the Arabidopsis DIMINUTO gene which has been shown to regulate cell elongation (Takahashi et al. 1995). Vigun11g191300 is a strong candidate as seed size is influenced by multiple pathways.

In summary, combined GWAS meta-analysis and comparative genomics have led to a better understanding of the genetic basis of seed size-related traits. QTLs harboring candidate genes have been identified which deserve further in-depth studies to explore their possible roles in cowpea seed size. A direct result of the present study has been to establish genetic markers of these variants, which now are available to facilitate cowpea breeding for improved varieties with a range of seed sizes.

Author contribution statement SL, MMA, SAH, SX, and TJC designed the study. SL analyzed the data with help from MMA, SAH, ADF, and SX. SL wrote the paper with inputs from SX, SAH, and TJC. MMA, TJC, PAR, NC read and reviewed the paper.

Acknowledgements This work was supported by grants from the Feed the Future Innovation Lab for Climate Resilient Cowpea (Cooperative Agreement AID-OAA-A-13-00070) and the NSF BREAD project "Advancing the Cowpea Genome for Food Security" (Award \#1543963). We thank Dr. Tiantian Zhu for her inputs, and Yi-Ning Guo and Audrey pongs for technical assistance. We also thank Dr. Stefano Lonardi and Steve Wanamaker (University of California Riverside, USA) for assistance with the cowpea genome sequence and annotations.

Open Access This article is distributed under the terms of the Creative Commons Attribution 4.0 International License (http://creativeco mmons.org/licenses/by/4.0/), which permits unrestricted use, distribution, and reproduction in any medium, provided you give appropriate credit to the original author(s) and the source, provide a link to the Creative Commons license, and indicate if changes were made.

\section{References}

Andargie M, Pasquet RS, Gowda BS, Muluvi GM, Timko MP (2014) Molecular mapping of QTLs for domestication-related traits in cowpea (Vigna unguiculata (L.) Walp.). Euphytica 200:401-412

Bartrina I, Jensen H, Novak O, Strnad M, Werner T, Schmülling T (2017) Gain-of-function mutants of the cytokinin receptors AHK2 and AHK3 regulate plant organ size, flowering time and plant longevity. Plant Physiol 173:1783-1797

Bjerkan KN, Jung-Roméo S, Jürgens G, Genschik P, Grini PE (2012) Arabidopsis WD repeat domain55 Interacts with DNA damaged binding protein 1 and is required for apical patterning in the embryo. Plant Cell 24:1013-1033

Burridge JD, Schneider HM, Huynh B-L, Roberts PA, Bucksch A, Lynch JP (2017) Genome-wide association mapping and agronomic impact of cowpea root architecture. Theor Appl Genet 130:419-431

Chapin FS III, Autumn K, Pugnaire F (1993) Evolution of suites of traits in response to environmental stress. Am Nat 142:S78-S92

Cheng W-H, Taliercio EW, Chourey PS (1996) The miniature1 seed locus of maize encodes a cell wall invertase required for normal development of endosperm and maternal cells in the pedicel. Plant Cell 8:971-983

Cleary A, Farmer A (2017) Genome Context Viewer: visual exploration of multiple annotated genomes using microsynteny. Bioinformatics 34:1562-1564

Dadson R, Hashem F, Javaid I, Joshi J, Allen A, Devine T (2005) Effect of water stress on the yield of cowpea (Vigna unguiculata L. Walp.) genotypes in the Delmarva region of the United States. J Agron Crop Sci 191:210-217

Drabo I, Redden R, Smithson J, Aggarwal V (1984) Inheritance of seed size in cowpea (Vigna unguiculata (L.) Walp.). Euphytica 33:929-934

Evangelou E, Ioannidis JPA (2013) Meta-analysis methods for genomewide association studies and beyond. Nat Rev Genet 14:379

Fatokun CA, Menancio-Hautea DI, Danesh D, Young ND (1992) Evidence for orthologous seed weight genes in cowpea and mung bean based on RFLP mapping. Genetics 132:841-846

Folsom JJ, Begcy K, Hao X, Wang D, Walia H (2014) Rice Fertilization-Independent Endosperm1 Regulates Seed Size under Heat Stress by Controlling Early Endosperm Development. Plant Physiol 165:238-248

Herniter IA, Muñoz-Amatriaín M, Lo S, Guo Y-N, Close TJ (2018) Identification of candidate genes controlling black seed coat and pod tip color in Cowpea (Vigna unguiculata [L.] Walp). Genes Genomes Genet 8:3347-3355

Huynh BL, Ehlers JD, Huang BE, Muñoz-Amatriaín M, Lonardi S, Santos JR, Ndeve A, Batieno BJ, Boukar O, Cisse N (2018) A 
multi-parent advanced generation inter-cross (MAGIC) population for genetic analysis and improvement of cowpea (Vigna unguiculata L. Walp.). Plant J 93:1129-1142

Igea J, Miller EF, Papadopulos AS, Tanentzap AJ (2017) Seed size and its rate of evolution correlate with species diversification across angiosperms. PLoS Biol 15:e2002792

Ihaka R, Gentleman R (1996) R: a language for data analysis and graphics. J Comput Graph Stat 5:299-314

Jin Y, Ni D-A, Ruan Y-L (2009) Posttranslational elevation of cell wall invertase activity by silencing its inhibitor in tomato delays leaf senescence and increases seed weight and fruit hexose level. Plant Cell 21:2072-2089

Jofuku KD, Omidyar PK, Gee Z, Okamuro JK (2005) Control of seed mass and seed yield by the floral homeotic gene APETALA2. Proc Natl Acad Sci USA 102:3117-3122

Kang EY, Han B, Furlotte N, Joo JWJ, Shih D, Davis RC, Lusis AJ, Eskin E (2014) Meta-analysis identifies gene-by-environment interactions as demonstrated in a study of 4965 mice. PLoS Genet 10:e1004022

Lo S, Muñoz-Amatriaín M, Boukar O, Herniter I, Cisse N, Guo Y-N, Roberts PA, Xu S, Fatokun C, Close TJ (2018) Identification of QTL controlling domestication-related traits in cowpea (Vigna unguiculata L. Walp). Sci Rep 8:6261

Lonardi S, Muñoz-Amatriaín M, Liang Q, Shu S, Wanamaker SI, Lo S, Tanskanen J, Schulman AH, Zhu T, Luo M-C, Alhakami H, Ounit R, Hasan AM, Verdier J, Roberts PA, Santos JRP, Ndeve A, Doležel J, Vrána J, Hokin SA, Farmer AD, Cannon SB, Close TJ (2019) The genome of cowpea (Vigna unguiculata [L.] Walp.). Plant J 98:767-782

Lucas MR, Huynh BL, da Silva Vinholes P, Cisse N, Drabo I, Ehlers JD, Roberts PA, Close TJ (2013) Association studies and legume synteny reveal haplotypes determining seed size in Vigna unguiculata. Front Plant Sci 4:95

Lush W, Wien H (1980) The importance of seed size in early growth of wild and domesticated cowpeas. J Agric Sci 94:177-182

Mishili FJ, Fulton J, Shehu M, Kushwaha S, Marfo K, Jamal M, Kergna A, Lowenberg-DeBoer J (2009) Consumer preferences for quality characteristics along the cowpea value chain in Nigeria, Ghana, and Mali. Agribusiness 25:16-35

Muñoz-Amatriaín M, Mirebrahim H, Xu P, Wanamaker SI, Luo M, Alhakami H, Alpert M, Atokple I, Batieno BJ, Boukar O, Bozdag S, Cisse N, Drabo I, Ehlers JD, Farmer A, Fatokun C, Gu YQ, Guo Y-N, Huynh B-L, Jackson SA, Kusi F, Lawley CT, Lucas MR, Ma Y, Timko MP, Wu J, You F, Barkley NA, Roberts PA, Lonardi S, Close TJ (2017) Genome resources for climate-resilient cowpea, an essential crop for food security. Plant J 89:1042-1054

Muñoz-Amatriaín M, Lo S, Close TJ (unpublished)

Nordborg M, Weigel D (2008) Next-generation genetics in plants. Nature 456:720-723

Ohad N, Margossian L, Hsu Y-C, Williams C, Repetti P, Fischer RL (1996) A mutation that allows endosperm development without fertilization. Proc Natl Acad Sci 93:5319-5324

Pan L, Wang N, Wu Z, Guo R, Yu X, Zheng Y, Xia Q, Gui S, Chen C (2017) A high density genetic map derived from RAD sequencing and its application in QTL analysis of yield-related traits in Vigna unguiculata. Front Plant Sci 8:1544

Riefler M, Novak O, Strnad M, Schmülling T (2006) Arabidopsis cytokinin receptor mutants reveal functions in shoot growth, leaf senescence, seed size, germination, root development, and cytokinin metabolism. Plant Cell 18:40-54

Rudner L, Glass GV, Evartt DL, Emery PJ (2002) A user's guide to the meta-analysis of research studies. ERIC Clearinghouse on Assessment and Evaluation, University of Maryland, College Park, p 37

Schmutz J, McClean PE, Mamidi S, Wu GA, Cannon SB, Grimwood J, Jenkins J, Shu S, Song Q, Chavarro C (2014) A reference genome for common bean and genome-wide analysis of dual domestications. Nat Genet 46:707

Singh B (2014) Cowpea: the food legume of the 21st century. Crop Science Society of America, Fitchburg

Takahashi T, Gasch A, Nishizawa N, Chua N-H (1995) The DIMINUTO gene of Arabidopsis is involved in regulating cell elongation. Genes Dev 9:97-107

van Nocker S, Ludwig P (2003) The WD-repeat protein superfamily in Arabidopsis: conservation and divergence in structure and function. BMC Genomics 4:50

Venglat P, Xiang D, Wang E, Datla R (2014) Genomics of seed development: challenges and opportunities for genetic improvement of seed traits in crop plants. Biocatal Agric Biotechnol 3:24-30

Wang M, Xu S (2019) Statistical power in genome-wide association studies and quantitative trait locus mapping. Heredity. https://doi. org/10.1038/s41437-019-0205-3

Wang A, Garcia D, Zhang H, Feng K, Chaudhury A, Berger F, Peacock WJ, Dennis ES, Luo M (2010) The VQ motif protein IKU1 regulates endosperm growth and seed size in Arabidopsis. Plant J 63:670-679

Wang X, Li Y, Zhang H, Sun G, Zhang W, Qiu L (2015) Evolution and association analysis of GmCYP78A10 gene with seed size/weight and pod number in soybean. Mol Biol Rep 42:489-496

Weber H, Borisjuk L, Wobus U (1996) Controlling seed development and seed size in Vicia faba: a role for seed coat-associated invertases and carbohydrate state. Plant J 10:823-834

Xu P, Wu X, Muñoz-Amatriaín M, Wang B, Wu X, Hu Y, Huynh BL, Close TJ, Roberts PA, Zhou W (2017) Genomic regions, cellular components and gene regulatory basis underlying pod length variations in cowpea (Vigna unguiculata L. Walp). Plant Biotechnol J 15:547-557

Zhang Z, Ersoz E, Lai C-Q, Todhunter RJ, Tiwari HK, Gore MA, Bradbury PJ, Yu J, Arnett DK, Ordovas JM (2010) Mixed linear model approach adapted for genome-wide association studies. Nat Genet 42:355-360

Zhao B, Dai A, Wei H, Yang S, Wang B, Jiang N, Feng X (2016) Arabidopsis KLU homologue GmCYP78A72 regulates seed size in soybean. Plant Mol Biol 90:33-47

Zhou Y, Zhang X, Kang X, Zhao X, Zhang X, Ni M (2009) SHORT HYPOCOTYL UNDER BLUE1 associates with MINISEED3 and HAIKU2 promoters in vivo to regulate Arabidopsis seed development. Plant Cell 21:106-117

Zhu C, Gore M, Buckler ES, Yu J (2008) Status and prospects of association mapping in plants. Plant Genome 1:5-20

Publisher's Note Springer Nature remains neutral with regard to jurisdictional claims in published maps and institutional affiliations. 